\title{
A CHAIN RULE FOR DIFFERENTIATION WITH APPLICATIONS TO MULTIVARIATE HERMITE POLYNOMIALS
}

\author{
C.S. WI THERS
}

\begin{abstract}
A chain rule is given for differentiating a multivariate function of a multivariate function. In the univariate case this chain rule reduces to Faa de Bruno's formula.

Using this, a simple procedure is given to obtain the rth order multivariate Hermite polynomial from the rth order univariate Hermite polynomial.
\end{abstract}

\section{The chain rule}

The following formula for the derivatives of a function of a function is easily verified.

$$
\text { For } f: R^{c} \rightarrow R^{d}, g: R^{d} \rightarrow R^{e}, \pi=\left(\alpha_{1}, \ldots, \alpha_{r}\right) \text { a set of } r
$$

integers in $\{1,2, \ldots, c\}$ and $x$ in $R^{\mathcal{C}}$, set $y=f(x)$, and $(f)_{\pi}(x)=\partial^{r} f(x) / \partial x_{\alpha_{1}} \ldots \partial x_{\alpha_{r}}$; then $(g \circ f)(x)=g(f(x))$ has $r$ th order derivatives

$$
(g \circ f)_{\pi}(x)=\sum_{k=1}^{r}(g)_{i_{1}} \ldots i_{k}(y) \sum_{\pi}\left(f_{i_{1}}\right)_{\pi_{1}}(x) \ldots\left(f_{i_{k}}\right)_{\pi_{k}}(x)
$$

where summation as each $i_{1}, \ldots, i_{k}$ ranges over 1 to $d$ is implicit,

Received 3 April 1984.

Copyright Clearance Centre, Inc. Serial-fee code: 0004-9727/84 $\$$ A2 $.00+0.00$. 
and $\sum_{\pi}$ sums over all partitions $\left(\pi_{1}, \ldots, \pi_{k}\right)$ of $\pi$.

If the number of sets $\pi_{1}, \ldots, \pi_{k}$ of length $i$ is $n_{i}$, $1 \leq i \leq r$, then $n_{1}+2 n_{2}+\ldots+r n_{r}=r, n_{1}+\ldots+n_{r}=k$, and the number of such partitions is $m(n)=r ! / \prod_{i=1}^{r}\left(i !{ }^{n} n_{i} !\right)$. Hence we may write $\sum_{\pi}=\sum_{n} \sum^{m(n)}$, where $\sum^{m(n)}$ sums over all $m(n)$ such partitions of $\pi$ and $\sum_{n}$ sums over all such $n$.

EXAMPLE. If $r=4$, the possibilities are

$\begin{array}{ccccccc}n_{1} & n_{2} & n_{3} & n_{4} & k & m(n) \\ 0 & 0 & 0 & 1 & 1 & 1 & \\ 1 & 0 & 1 & 0 & 2 & 4 & \\ 0 & 2 & 0 & 0 & 2 & 3 & \\ 2 & 1 & 0 & 0 & 3 & 6 & \\ 4 & 0 & 0 & 0 & 4 & 1 & ;\end{array}$

hence

$$
\begin{aligned}
& (g \circ f)_{\alpha_{1} \ldots \alpha_{4}}(x)=(g)_{i_{1}}\left(f_{i_{1}}\right)_{\alpha_{1} \ldots \alpha_{4}} \\
& +(g)_{i_{1} i_{2}}\left\{\sum^{4}\left(f_{i_{1}}\right)_{\alpha_{1}}\left(f_{i_{2}}\right)_{\alpha_{2} \alpha_{3} \alpha_{4}}+\sum^{3}\left(f_{i_{1}}\right)_{\alpha_{1} \alpha_{2}}\left(f_{i_{2}}\right)_{\alpha_{3} \alpha_{4}}\right\} \\
& +(g)_{i_{1} i_{2} i_{3}} \sum^{6}\left(f_{i_{1}}\right)_{\alpha_{1}}\left(f_{i_{2}}\right)_{\alpha_{2}}\left(f_{i_{3}}\right)_{\alpha_{3} \alpha_{4}} \\
& +(g)_{i_{1} i_{2} i_{3} i_{4}}\left(f_{i_{1}}\right)_{\alpha_{1}}\left(f_{i_{2}}\right)_{\alpha_{2}}\left(f_{i_{3}}\right)_{\alpha_{3}}\left(f_{i_{4}}\right)_{\alpha_{4}} \text {, }
\end{aligned}
$$

where $(g)_{\pi}=(g)_{\pi}(y)$ and $\left(f_{i}\right)_{\pi}=\left(f_{i}\right)_{\pi}(x)$.

If $c=d=1$ this becomes Faa de Bruno's formula

$$
(g \circ f)^{(r)}(x)=\sum_{k=1}^{r} g^{(k)}(y) \sum_{n} m(n) f^{(1)}(x)^{n} \ldots f^{(r)}(x)^{n} r
$$

where $f^{(r)}(x)=(d / d x)^{r} f(x) ;$ see Goursat [2, p. 34]. 


\section{The multivariate Hermite polynomials}

The rth univariate Hermite polynomial is defined as

$$
H e_{p}(x)=\exp \left(x^{2} / 2\right)(-d / d x)^{r} \exp \left(-x^{2} / 2\right), r \geq 0, x \text { in } R .
$$

The first 10 are given in Kendall and Stuart [3, p. 155]. For example,

$$
H_{5}(x)=x^{5}-10 x^{3}+15 x
$$

Let $A=\left(A_{i j}\right)$ be any symmetric $c \times c$ matrix, $x$ any point in $R^{f}$, and $\alpha=\left(\alpha_{1}, \ldots, \alpha_{p}\right)$, where for $1 \leq i \leq r, \alpha_{i}$ is any number in $\{1,2, \ldots, c\}$. Then the general $r$ th order Hermite polynomial is

$$
H e_{\alpha}(x, A)=(-)^{r} \exp (Q / 2)\left(\partial^{r} / \partial x_{\alpha_{1}} \ldots \partial x_{\alpha_{p}}\right) \exp (-Q / 2) \text {, where } Q=x^{\prime} A x \text {. }
$$

These polynomials are the building block for multivariate Edgeworth expansions.

(For some results on these see Erdelyi [1, p. 285]; his notation is different.) An expression for $H_{\alpha}(x, A)$ follows immediately from that of $H e_{p}(x)$. This is best illustrated by an example. From $H e_{5}(x)$ above it follows that, for $r=5$,

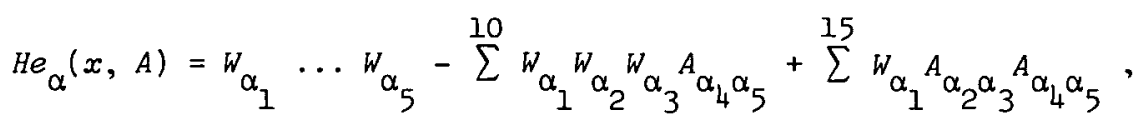

where

$$
w_{i}=\sum_{j=1}^{r} A_{i j} x_{j}
$$

and

$$
\sum^{m} w_{a_{1}} \cdots w_{a_{2} b_{1} b_{2}} \cdots A_{b_{2 k-1} b_{2 k}}=\sum^{m} I_{\eta, 2 k}(a, b)
$$

say, denotes the sum over all $m=(2+2 k) ! /\left(2 ! 2^{k} k !\right)$ partitions $a, b$ of $\alpha$ of length $l$ and $2 k$ respectively, allowing for the symmetry of $A$. For example 


$$
\sum^{3} W_{a b c} A_{b c}=W_{a} A_{b c}+W_{b} A_{c a}+W_{c} A_{a b}
$$

The general formula

$$
H e_{\alpha}(x, A)=\sum_{z+2 k=r}(-)^{k} \sum^{m} I_{\eta, 2 k}(a, b)
$$

follows from $\S 1$.

\section{References}

[1] A. Erdelyi, Higher transcendental functions, volume 2 (McGraw-Hill, New York, 1953).

[2] E. Goursat, A course in mathematical analysis, volume 1 (Dover, New York, 1959).

[3] M.G. Kendall and A. Stuart, The advanced theory of statistics, volume 1 , second edition (Griffin, London, 1963).

Applied Mathematics Division,

DSIR,

Box 1335,

Wellington,

New Zealand. 